Macgregor, S., Visscher, P. M., Knott, S., et al (2002) Is schizophrenia linked to chromosome lq? Science, 298 2277.

Ross, C. A., Margolis, R. L., Reading, S. A. J., et al (2006) Neurobiology of schizophrenia. Neuron, 52, 139153.

W. J. Muir Division of Psychiatry, University of Edinburgh, Kennedy Tower, Royal Edinburgh Hospital, Morningside Park, Edinburgh EHI0 5HF, UK. Email: walter.muir@ed.ac.uk

B. S. Pickard Medical Genetics Section, University of Edinburgh, Edinburgh, UK

D. H. Blackwood Division of Psychiatry, School of Molecular and Clinical Medicine, University of Edinburgh, Edinburgh, UK

doi: 10.I192/bjp.190.3.270a

\section{Hippocampal and amygdala volume reductions in first-episode schizophrenia}

Steen et al (2006) performed a systematic review and meta-analysis of cross-sectional and longitudinal magnetic resonance imaging (MRI) studies of brain volumes in patients with first-episode psychosis and healthy controls. Despite some methodological differences, the findings were in line with a recent meta-analysis performed by our group (Vita et al, 2006).

A significant decrease in hippocampal but not amygdala volumes was found in patients at illness onset in both reviews. Another relevant paper reporting amygdala and hippocampal volumes in a large sample of patients with first-episode schizophrenia was published after these two meta-analyses (Velakoulis et al, 2006). Thus we considered it worthwhile to conduct a new set of meta-analyses including these MRI data.

The results of the new meta-analyses for hippocampus (7 studies, 290 patients, 355 controls) and amygdala (5 studies, 218 patients, 175 controls) confirmed our previous findings. Even with the inclusion of the study of Velakoulis et al (2006), the composite effect sizes for the hippocampus remained significant $(d=0.357,95 \%$ CI $0.208-0.541$ for the right hippocampus and $0.574,95 \%$ CI $0.405-0.742$ for the left hippocampus) whereas those for the amygdala were not $(d=-0.046,95 \%$ CI -0.247 to 0.154 for the right amygdala and $0.025,95 \%$ CI -0.175 to 0.226 for the left amygdala).

These results, in line with those of Steen et al (2006), support the hypothesis of different patterns of involvement of temporolimbic structures over the course of schizophrenia, with the hippocampus affected earlier than the amygdala. In our opinion, these findings have important implications for future neurobiological studies of schizophrenia and emphasise the importance of longitudinal studies to address the issue of different times of occurrence and progression of brain abnormalities in people with first-episode schizophrenia.

Steen, R. G., Mull, C., McClure, R. M., et al (2006) Brain volume in first-episode schizophrenia. Systematic review and meta-analysis of magnetic resonance imaging studies. British Journal of Psychiatry, 188, 510-518.

Velakoulis, D., Wood, S. J., Wong, M. T., et al (2006) Hippocampal and amygdala volumes according to psychosis stage and diagnosis: a magnetic resonance imaging study of chronic schizophrenia, first-episode psychosis, and ultra-high-risk individuals. Archives of General Psychiatry, 63, 139-149.

Vita, A., De Peri, L., Silenzi, C., et al (2006) Brain morphology in first-episode schizophrenia. A metaanalysis of quantitative magnetic resonance imaging studies. Schizophrenia Research, 82, 75-88.

A.Vita Psychiatric Unit, University of Brescia, Brescia, Italy

L. de Peri University of Milan, Via Francesco Sforza n. 35, Milan 20122, Italy. Email:

luca_de_peri@libero.it

doi: 10.1192/bjp.190.3.27|

\section{Effectiveness of cognitive- behavioural intervention by mental health nurses in schizophrenia}

Turkington et al (2006) report on outcomes of an effectiveness trial of brief cognitivebehavioural therapy (CBT) by mental health nurses in schizophrenia. Unfortunately there are flaws in the methodology, which casts major doubts on the validity of the study (Quitkin et al, 2000). First, although the authors claim to have a control group, it seems that patients in the control group did not have a placebo-like intervention; for example, the nurses could have spent the same amount of time with the patients without providing the CBT intervention. What is more surprising is that the study was powered to give a $90 \%$ chance of detecting only a $25 \%$ level difference in overall symptoms at the 0.01 level of significance. A $25 \%$ difference between a treatment and non-intervention group can easily be accounted for by a placebo effect. It is well known that the placebo response rate is usually around $30 \%$ in psychiatric trials. For over 50 years the inclusion of a placebo control group has been the standard for determining the efficacy of an intervention. Without an adequate comparison group and without adequate comparison conditions, it is impossible to differentiate any specific effects from other 'non-specific' factors, including chance variation, regression to the mean, healthcare provider attention, treatment credibility and rationale, persuasion, patient expectancy effects, researcher allegiance effects, effort justification, spontaneous remission, demand characteristics, etc. (Lohr et al, 1999).

Given the lack of a true control group this study would be called nothing but an open-label trial. Open-label trials require at least a $50 \%$ level difference in overall symptoms between baseline and post-intervention response; moreover they do not require huge numbers of patients to show a tendency towards improvement.

Lohr, J. M., Lilienfeld, S. O., Tolin, D. R., et al (1999) Eye movement desensitization and reprocessing: an analysis of specific versus nonspecific treatment factors. Journal of Anxiety Disorders, 13, 185-207.

Quitkin, F. M., Rabkin, J. G., Gerald, J., et al (2000) Validity of clinical trials of antidepressants. American Journal of Psychiatry, I57, 327-337.

Turkington, D., Kingdon, D., Rathod, S., et al (2006) Outcomes of an effectiveness trial of cognitivebehavioural intervention by mental health nurses in schizophrenia. British Journal of Psychiatry $189,36-40$.

F. Alam Avondale Unit, Royal Preston Hospital, Sharoe Green Lane, Preston PR2 9HT, UK. Email: docftalam@aol.com

doi: 10.I192/bjp.190.3.27la

Authors' reply: We believe that Dr Alam has misunderstood the difference between efficacy and effectiveness research. The national guidelines on the clinical management of schizophrenia (National Institute for Clinical Excellence, 2002) confirmed CBT to be an evidence-based treatment for persistent symptoms of schizophrenia. However, that decision was based almost entirely on efficacy trials where CBT was given by expert therapists to highly selected samples of people with schizophrenia without comorbidities and using an active comparator such as befriending or supportive counselling (e.g. Sensky et al, 2000). Expert therapists and uncomplicated patients are 